\title{
Flagellin influences the expression of a variety of important cytokines and chemokines without affecting the immune status of umbilical cord mesenchymal stem cells
}

\author{
QUANSHENG $\mathrm{LI}^{1}, \mathrm{YU} \mathrm{MA}^{2}, \mathrm{LI} \mathrm{LI}^{3}, \mathrm{JI} \mathrm{BAO}^{3}$ and $\mathrm{LI} Z \mathrm{ZHANG}^{3}$ \\ Departments of ${ }^{1}$ Biliary Surgery and ${ }^{2}$ Thyroid and Breast Surgery; ${ }^{3}$ Laboratory of Pathology, Department of Pathology, \\ West China Hospital, Sichuan University, Chengdu, Sichuan 610041, P.R. China
}

Received November 14, 2014; Accepted July 22, 2015

DOI: $10.3892 / \mathrm{mmr} .2015 .4276$

\begin{abstract}
Multipotent differentiation ability, low expression of co-stimulatory molecules and immunosuppressive effects render mesenchymal stem cells (MSCs) the best candidate for use in cell-based therapy. At present, MSCs are widely used in clinical trials, which have, however, failed to demonstrate any long-term therapeutic benefits of MSCs. Therefore, to improve the therapeutic potential of MSCs, it is important to understand the underlying mechanisms of the rapid disappearance of MSCs after transplantation. Toll-like receptors (TLRs) have important roles in the induction of the immune response and the regulation of biological functions. The present study employed TLR5 agonist flagellin in order to assess whether the activation of the TLR5 pathway alters the immune status and influences the differentiation ability of MSCs isolated from umbilical cords (UC). Assessment of the proliferation of peripheral blood mononuclear cells co-cultured with UCMSCs as well as their lactate dehydrogenase release indicated that flagellin stimulation failed to increase the immunogenicity of UCMSCs. Flow cytometric and histological cell differentiation analyses also confirmed that TLR5 activation neither enhanced the expression of co-stimulatory molecules (CD80, CD86 and human leukocyte antigen-E), nor influenced the differentiation ability of UCMSCs. However, polymerase chain reaction analysis indicated the induction of pro-inflammatory molecules by flagellin, which suggested that the induced immune-associated molecules may mediate biological functions of UCMSCs other than those assessed by the present study. Future studies
\end{abstract}

Correspondence to: Professor Li Zhang, Laboratory of Pathology, Department of Pathology, West China Hospital, Sichuan University, 37 Guoxue Road, Chengdu, Sichuan 610041, P.R. China

E-mail: zhangli7375@scu.edu.cn

Key words: mesenchymal stem cells, umbilical cord, toll-like receptor 5, flagellin, immunogenicity should focus on the role of TLR5 in regulating other functions of UCMSCs.

\section{Introduction}

Mesenchymal stem cells (MSCs) were initially isolated from bone marrow (BM) but are now known to reside in almost every type of connective tissue. MSCs are a heterogeneous subset of cells which can proliferate in vitro as plastic-adherent cells to develop as fibroblast colony-forming units (1). The most important characteristics of MSCs are their self-renewal and differentiation ability (2). Other important features of MSCs include the lack of co-stimulator molecules [CD80, CD86 and human leukocyte antigen (HLA)-E] (3) and immunosuppressive effects (4). All of these properties render MSCs as the best candidate for use in cell-based therapy. MSCs have now been widely evaluated in clinical trials on the treatment of conditions including left ventricular ejection fraction, graft versus host disease, pancreatic regeneration and rheumatoid arthritis (5). However, the results of clinical trials have not met their primary aims, and the current understanding of the fate of MSC following systemic infusion is limited (6). Previous studies indicated an enhanced immunogenicity of MSCs under specific conditions or upon specific stimulation, as indicated by changes in their differentiation status (7), as well as the activation of the memory $\mathrm{T}$ cell response (8) and the complement response (9).

Toll-like receptors (TLRs) are type I integral membrane glycoproteins known as pathogen-associated molecular patterns (PAMPs). The human TLR family has 11 members, which all have important roles in recognizing microbial components (10). It has also been indicated that TLRs are important in regulating the biological functions of MSCs isolated from BM and adipose tissues (11). However, to date, no study has elucidated the role of TLRs in influencing the immunogenicity of MSCs isolated from umbilical cords (UCs). Our group has performed systematic investigations to confirm the role of TLRs in promoting the immunogenicity of MSCs from UCs, which indicated that the activation of TLR7 enhances the immunogenicity of UCMSCs (12). In the present study, the TLR5 pathway was stimulated in UCMSCs by flagellin treatment and the effects of the activation of TLR5 on the immunogenicity of UCMSCs were investigated. 
Table I. Primers used for real-time polymerase chain reaction analysis.

\begin{tabular}{lllc}
\hline Gene & \multicolumn{1}{c}{ Forward primer (5'-3') } & \multicolumn{1}{c}{ Reverse primer (5'-3') } & GenBank number \\
\hline IL-1 $\beta$ & ACGAATCTCCGACCACCACT & CCATGGCCACAACAACTGAC & M15330 \\
IL-6 & GACCCAACCACAAATGCCA & GTCATGTCCTGCAGCCACTG & M14584 \\
IL-8 & CTGGCCGTGGCTCTCTTG & CCTTGGCAAAACTGCACCTT & NM_000584 \\
IL-10 & GGTGATGCCCCAAGTGA & TCCCCCAGGGAGTTCACA & U16720 \\
IL-12 & CGGTCATCTGCCGCAAA & CAAGATGAGCTATAGTAGCGGTCCT & M65272 \\
IFN- $\beta$ & CAGCAATTTCAGTGTCAGAAGCT & TCATCCTGTCCTTGAGGCAGT & M28622 \\
IP-10 & TGAAATTATTCCTGCAAGCCAA & CAGACATCTCTTCTCACCCTTCTTT & NM_001565 \\
NF- B & AGAGTGCTGGAGTTCAGGATA & AAGGTGGATGATTGCTAAGTGT & AJ271718 \\
TGF- $\beta$ & TATCGACATGGAGCTGGTGAAG & CAGCTTGGACAGGATCTGGC & X02812 \\
TNF- $\alpha$ & GGTGCTTGTTCCTCAGCCTC & CAGGCAGAAGAGCGTGGTG & M10988 \\
CCL5 & AGCAGAGGCTGGAGAGCTACA & GGGTCAGCACAGATCTCCTTGT & NM_006273 \\
CCL24 & CCTCTCCTGCCTCATGCTTATT & CTCTGTCTCTGCATCATTTGTGAA & U58914 \\
GAPDH & GAAGGTGAAGGTCGGAGTC & GAAGATGGTGATGGGATTTC & J04038 \\
\hline
\end{tabular}

\section{Materials and methods}

Isolation and culture of UCMSCs. The UCMSCs (Sichuan Umbilical Cord Blood Stem Cell Bank, Sichuan, China) were isolated from two UCs as previously reported (13). After disinfection in $75 \%$ ethanol for $20 \mathrm{~min}$, the UC was dissected into cubical-shaped pieces, which were planted on $25 \mathrm{~mm}^{2}$ plates. Complete Dulbecco's modified Eagle's medium (DMEM; Invitrogen Life Technologies, Carlsbad, CA, USA) with $10 \%$ fetal bovine serum (Invitrogen Life Technologies) was added to the plates with the small UC tissues tightly attached to the bottom. The tissues were removed when dissociated MSCs were observed following culture for almost eight days. The UCMSCs were then digested with trypsin (Invitrogen Life Technologies) and either stored in liquid nitrogen for future use or subjected to further culturing. The present study was approved by the ethics committee of the West China Hospital, Sichuan University (Chengdu, China), and written informed consent was obtained from the patients.

TLR5 stimulation. TLR5 agonist flagellin (no. AG-40B-0025; Adipogen, San Diego, CA, USA) was dissolved in sterile water at a concentration of $0.1 \mathrm{mg} / \mathrm{ml}$. UCMSCs were seeded into a six-well plate at a density of $1.5 \times 10^{5}$ in $2 \mathrm{ml}$ medium and flagellin was added to the medium at a final concentration of $50 \mathrm{ng} / \mathrm{ml}$.

Assessment of leukocyte proliferation. Peripheral blood mononuclear cells (PBMCs) were obtained from two healthy student volunteers were recruited from the Laboratory of Pathology, West China Hospital, Sichuan University, and isolated by density centrifugation gradient (300 x g, $20 \mathrm{~min})$ and labeled by carboxyfluorescein diacetate succinimidyl ester (CFSE; eBioscience, San Diego, CA, USA) at a final concentration of $10 \mu \mathrm{M}$. Pre-cooled complete DMEM was added following $10 \mathrm{~min}$ to terminate the reaction. The CFSE-labeled PBMCs were then washed three times with cold phosphate-buffered saline (1,200 xg, 5-min). PBMCs were cultured alone or co-cultured with UCMSCs (10:1) in
Table II. Monoclonal antibodies used for fluorescence-assisted cell sorting analysis.

\begin{tabular}{lc}
\hline Name & Catalog number \\
\hline CD80 & $11-0809$ \\
CD86 & $12-0869$ \\
HLA-E & $17-9953$ \\
CD90 & $45-0909$ \\
CD59 & $11-0596$ \\
CD29 & $17-0299$ \\
\hline
\end{tabular}

the absence or presence of flagellin for $72 \mathrm{~h}$ and collected for fluorescence-associated cell sorting (FACS) detection (FACScan flow cytometer; Beckman Coulter, Brea, CA, USA).

Lactate dehydrogenase $(\mathrm{LDH})$ assay. Supernatants from the PBMC-UCMSC co-culture system were collected at 24, 48 and $72 \mathrm{~h}$ post-stimulation and assessed using the cytotoxicity detection LDH kit (cat. no. G1780; Promega Corporation, Madison, WI, USA). The release of LDH from damaged cells was assayed according to the manufacturer's instructions. Colorimetric evaluation was conducted using a MQX200 plate reader, BioTek, Winooski, VT, USA. Percentage of lysed cells was calculated by the following formula: $(\mathrm{E}-\mathrm{M}) /(\mathrm{T}-\mathrm{M}) \times 100 \%$, where $\mathrm{E}$ is experimental release, $\mathrm{M}$ is the spontaneous release in the presence of media alone, and $\mathrm{T}$ is the maximum release in the presence of $5 \%$ Triton X-100 (Invitrogen Life Technologies).

Flow cytometric analysis of surface markers. Flagellin-treated and untreated UCMSCs were harvested $72 \mathrm{~h}$ post-stimulation and stained with antibodies (eBioscience; 1:100 dilution; incubated for $30 \mathrm{~min}$ at room temperature) against surface marker molecules (Table II). Flow cytometric results were analyzed using CXP flow cytometry software 
A

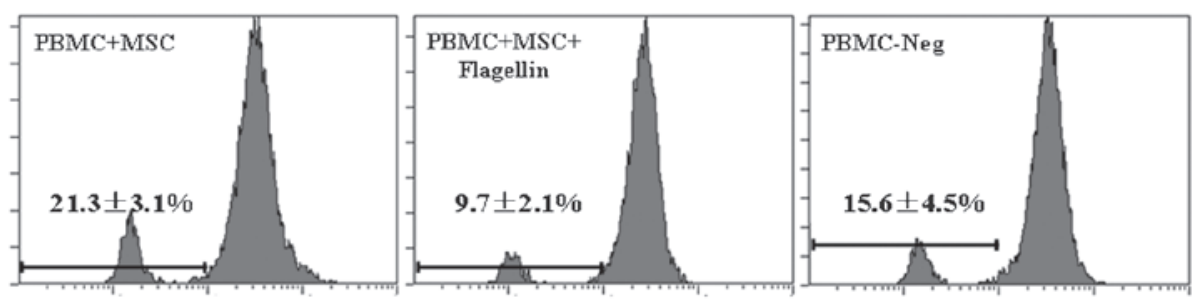

B $(\%)$

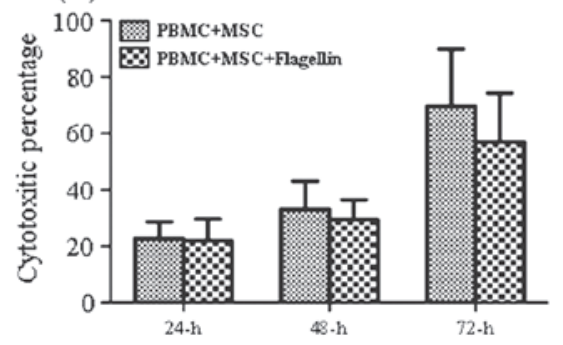

Figure 1. PBMC proliferation and LDH release in a PBMC-UCMSC co-culture system. (A) Co-culture of PBMC and UCMSC; the percentage of PBMCs is shown. (B) Detection of LDH for the assessment of cytotoxicity. Values are expressed as the mean \pm standard error of the mean from three experiments with similar results. PBMC, peripheral blood mononuclear cells; UCMSC, umbilical cord mesenchymal stem cells; LDH, lactate dehydrogenase.

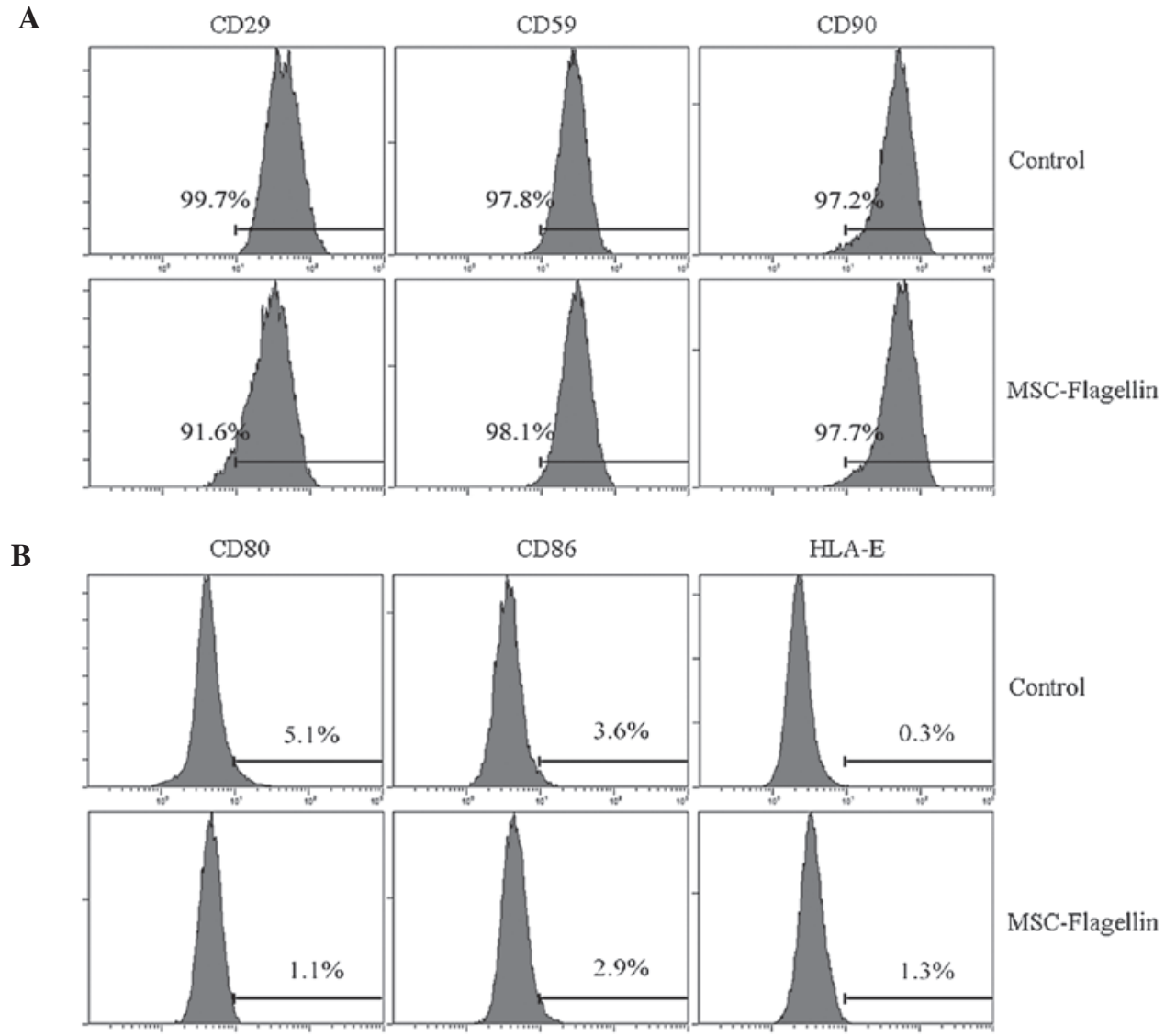

Figure 2. Analysis of surface markers and co-stimulatory molecules in umbilical cord mesenchymal stem cells upon flagellin stimulation. (A) Surface stem cell markers and (B) co-stimulatory molecules. The results are representative of three experiments with similar results. HLA, human leukocyte antigen.

(Beckman Coulter). The positive standard of cells was gated according to the fluorescence intensity of the negative control group.

UCMSC differentiation. UCMSCs were seeded into six-well plates at $1.5 \times 10^{5}$ cells per well. Differentiation medium specific for chondrocytes (no. A10071-01; Gibco-BRL,
Invitrogen Life Technologies), osteocytes (no. A10072-01; Gibco-BRL) and adipocytes (no. A10070-01; Gibco-BRL) was added to the respective wells together with $10 \mu \mathrm{g} / \mathrm{ml}$ imiquimod (Novus Biologicals, LLC, Littleton, CO, USA). Oil-red O (eBioscience) was used for staining of adipocytes, alizarin red (eBioscience) for osteocytes and safranine (eBioscience) for chondrocytes at 7, 14 and 21 days of culture. 
A
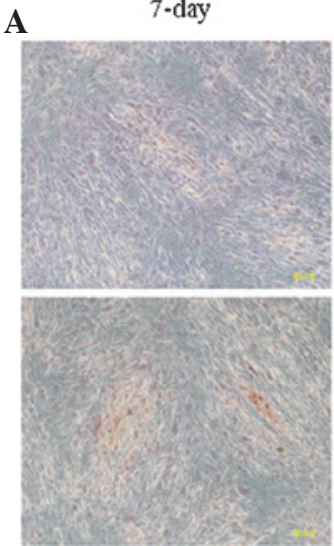

B

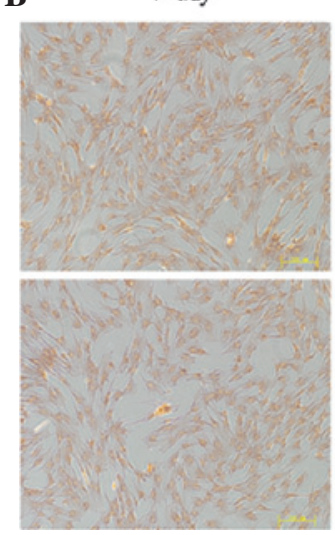

C
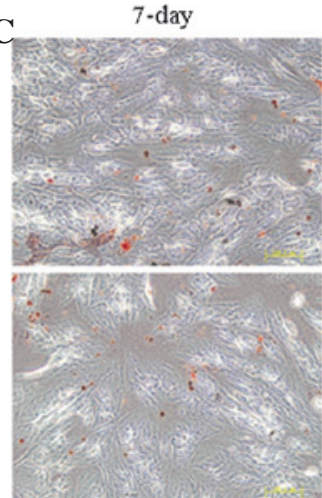

14-day
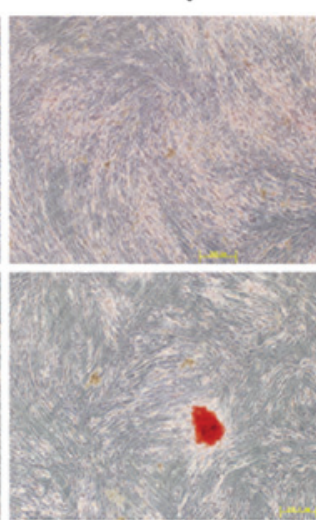

14-day
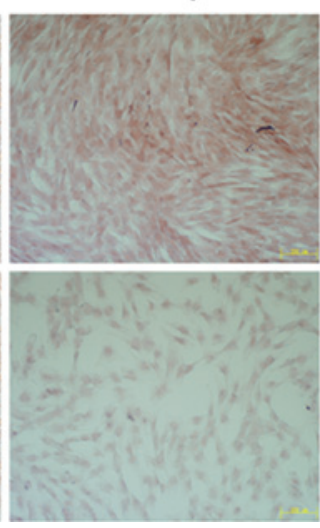

14-day

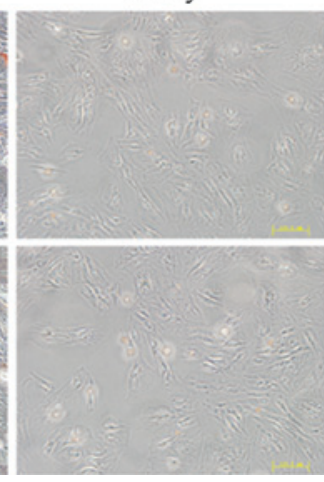

21-day

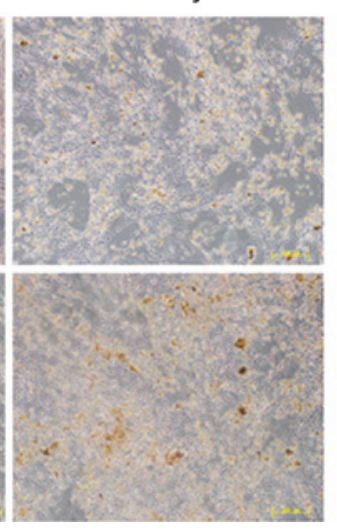

MSC-control

MSC+Flagellin

21-day

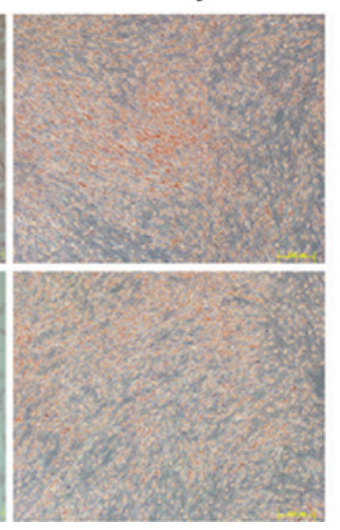

MSC-control

MSC+Flagellin

21-day

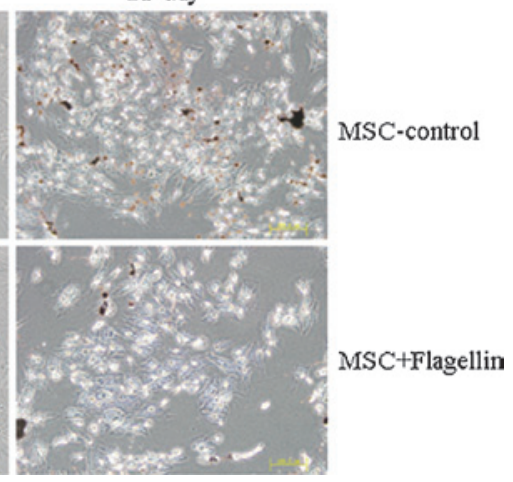

Figure 3. Differentiation measure of umbilical cord MSCs upon flagellin stimulation. (A) Osteoblast differentiation (stain: Alizarin red; x100 magnification), (B) chondrocyte differentiation (stain: Safranin; x100 magnification) and (C) adipocyte differentiation (stain: Oil red O; x200 magnification). The results are representative of three experiments with similar results. Scale bar, $50 \mu \mathrm{m}$. MSC, mesenchymal stem cell.

Reverse transcription quantitative polymerase chain reaction $(R T-q P C R)$. The RNA extraction, cDNA synthesis and quantitative PCR were performed according to a protocol of our previous study (12). After treatment with flagellin $(50 \mathrm{ng} / \mathrm{ml})$ for $4,12,24,72$ or $120 \mathrm{~h}$, total RNA was isolated from the UCMSCs using an RNeasy mini kit (Qiagen, Hilden, Germany). cDNA was synthesized from 50 ng RNA using the ReverTra Ace qPCR kit (FSQ-101; Toyobo, Osaka, Japan). The RT conditions were $65^{\circ} \mathrm{C}$ for $5 \mathrm{~min}$, followed by $37^{\circ} \mathrm{C}$ for $15 \mathrm{~min}$ and $98^{\circ} \mathrm{C}$ for $5 \mathrm{~min}$. qPCR was performed using RealMaster mix (SYBR Green; FP202; Tiangen, Beijing, China) with 50 ng cDNA. qPCR was performed in an iCycler iQTM Optical Module (Beckman Coulter) under the following conditions: One cycle at $95^{\circ} \mathrm{C}$ for $30 \mathrm{sec}$, then 40 cycles at $95^{\circ} \mathrm{C}$ for $30 \mathrm{sec}, 58^{\circ} \mathrm{C}$ for $30 \mathrm{sec}$ and $72^{\circ} \mathrm{C}$ for $30 \mathrm{sec}$, followed by a melting curve from $55-95^{\circ} \mathrm{C}$ in $0.5^{\circ} \mathrm{C}$ increments and 10 -sec intervals. The primers used (Chengdu Branch Zi Qing Xi Biotechnology Co., Ltd., Chengdu, China) are listed in Table I. All experiments were performed three times.

Statistical analysis. The qPCR data were analyzed using Bio-Rad iQ5 software (Bio-Rad Laboratories, Inc., Hercules, CA, USA). GAPDH was used as an internal control. Normal peripheral blood lymphocytes were used as a negative control. Values are expressed as the mean \pm standard error of the mean. Statistical analyses were performed using SPSS 16.0 (SPSS, Inc., Chicago, IL, USA). Values of $\mathrm{P}<0.05$ and $\mathrm{P}<0.001$ were considered to indicate a significant difference compared with the control group. Graphs were prepared using GraphPad Prism5 (GraphPad Software, Inc., La Jolla, CA, USA). 


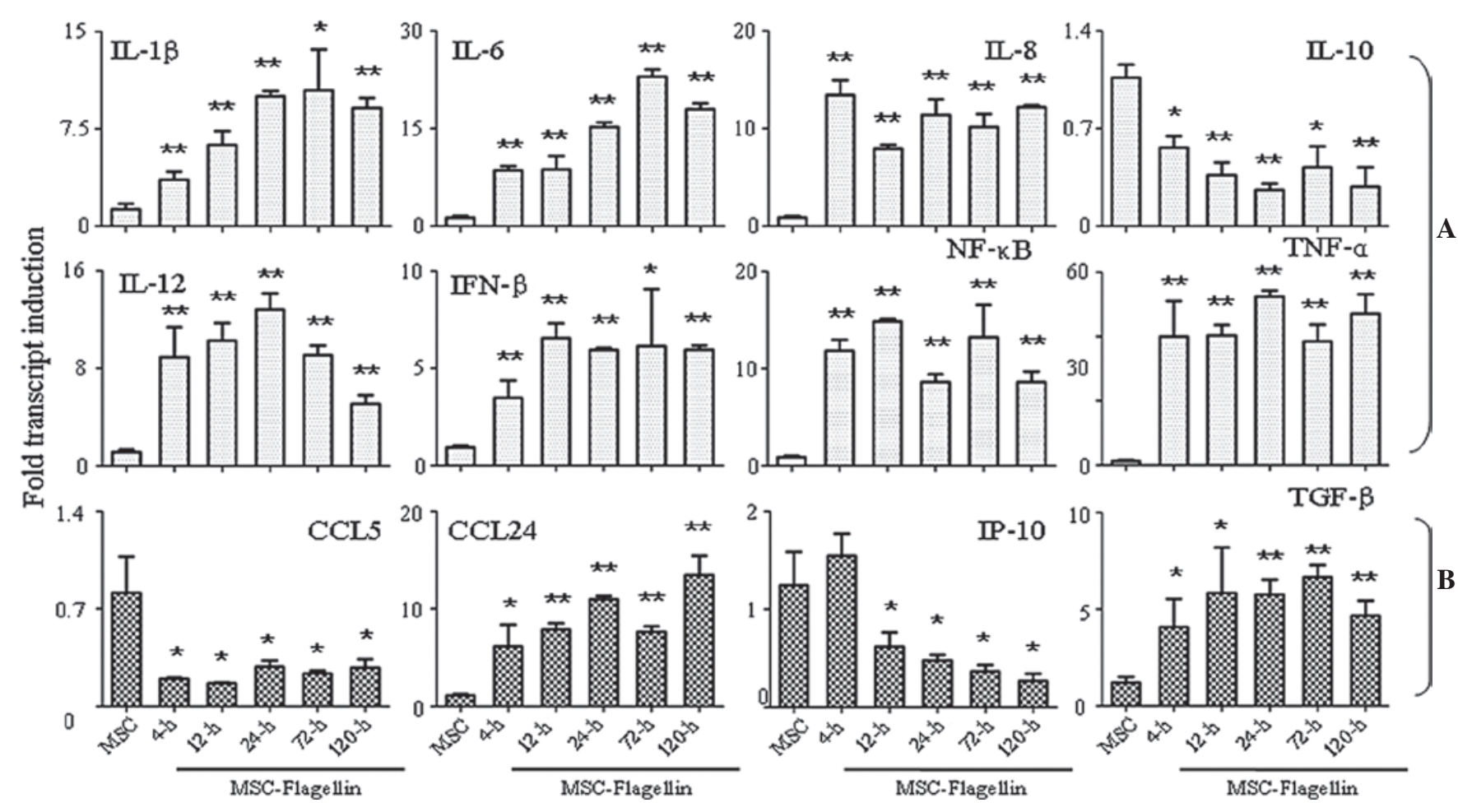

Figure 4. Flagellin promotes the expression of pro-inflammatory molecules as well as chemokines. (A) Pro-inflammatory molecules and (B) chemokines. Values are expressed as the mean \pm standard error of the mean of three independent experiments. ${ }^{* *} \mathrm{P}<0.001 ;{ }^{*} \mathrm{P}<0.05$ versus control group.

\section{Results}

TLR5 activation did not affect the proliferation of PBMCs or leukocyte-mediated cytotoxicity. PBMCs from human volunteers were labeled with CFSE and then co-cultured with UCMSCs in the presence of flagellin. PBMCs were collected and their proliferation was assessed by flow cytometric analysis following $72 \mathrm{~h}$ of co-culture. The results showed that the proliferation of PBMCs in the PBMC-UCMSC co-culture system in the presence of TLR5 agonist (9.7\%) was not obviously different from that in the control groups $(21.3 \%$ for PBMC-MSCs and $15.6 \%$ for PBMCs only), which suggested that TLR5 activation did not stimulate the immune response (Fig. 1A).

Leukocyte-mediated cytotoxicity was determined by measuring the LDH release by damaged cells in the culture supernatant. The results indicated that LDH levels were not significantly different between the experimental group (PBMC-UCMSCs co-culture in the presence of flagellin) and control group (PBMCs + MSCs) following 24, 48 and $72 \mathrm{~h}$ of incubation (Fig. 1B). This result indicated that the activation of the TLR5 pathway did not stimulate the in vitro immune response in the PBMC-UCMSC co-culture system.

TLR5 activation does not affect the expression of co-stimulatory molecules and stem cell markers. The observation that flagellin did not change the immune status motivated us to investigate whether the activation of TLR5 influences the expression of surface molecules on UCMSCs, particularly that of co-stimulatory molecules and stem cell markers. UCMSCs were incubated with or without flagellin, and the co-stimulatory molecules CD80, CD86 and HLA-E, as well as the surface stem cell markers CD29, CD59 and CD90 were assessed by RT-qPCR. The results showed that stimulation with flagellin for five days did not significantly alter the expression of the co-stimulatory factors $(\mathrm{P}>0.05$; $\mathrm{CD} 80,5.1$ vs. $1.1 \%$; CD86, 3.6 vs. $2.9 \%$; HLA-E, 0.3 vs. 1.3 ; control vs. flagellin group, respectively) (Fig. 2A). The expression of stem cell markers was also not significantly changed (CD29, 99.7 vs. 91.6\%; CD59, 97.8 vs. $98.1 \%$; CD90, 97.2 vs. $97.7 \%$; control vs. flagellin group, respectively) (Fig. 2B).

Activation of the TLR5 pathway does not affect the differentiation ability of UCMSCs. Next, the present study examined the role of the TLR5 pathway in regulating the differentiation of UCMSCs. Culture was performed in differentiation media specific for adipocytes, osteoblasts and chondrocytes to induce UCMSC differentiation (Fig. 3). The cell phenotypes were identified by staining with alizarin red for osteoblasts, safranine for chondrocytes and Oil red O for adipocytes in order to assess the differentiation levels of UCMSCs at 7, 14 and 21 days of culture in the presence of flagellin. The results clearly showed that there were no differences between the control and flagellin-treated groups, indicating that the TLR5 pathway was not involved in the differentiation of UCMSCs (Fig. 3A-C).

Activation of TLR5 enhances the expression of cytokines and chemokines. Finally, the present study performed an RT-qPCR analysis to assess the expression of several important cytokines and chemokines, which have significant roles in biological functions of UCMSCs. Eight important pro-inflammatory molecules (IL-1 $\beta$, IL-6, IL-8, IL-10, IL-12, IFN- $\beta$, NF- $k$ B and TNF- $\alpha$ ) were analyzed, which showed that the expression of all 
tested factors except IL-10 was markedly enhanced at 4, 12, 24, 72 and 120 h post-stimulation (all $\mathrm{P}<0.05$ or $\mathrm{P}<0.001$ ) (Fig. 4A). IL-10 expression was significantly inhibited following 4-h treatment with flagellin, which persisted for $120 \mathrm{~h}$ until the end of the experiment $(\mathrm{P}<0.05$ or $\mathrm{P}<0.001)$ (Fig. $4 \mathrm{~A})$. With regard to chemokine expression, CCL5 was inhibited in the presence of flagellin stimulation for 4-120 h $(\mathrm{P}<0.05)$, and IP-10 was also found inhibited following treatment for $12-120 \mathrm{~h}(\mathrm{P}<0.05)$ (Fig. 4B). The expression of the other two chemokines, CCL24 and TGF- $\beta$, was significantly induced upon flagellin stimulation $(\mathrm{P}<0.05$ and $\mathrm{P}<0.001)$ (Fig. 4B).

\section{Discussion}

MSCs can differentiate into mesodermal cells, including bone, cartilage, adipocyte and connective stromal cells. Furthermore, it was evidenced that MSCs differentiate into not only ectodermal lineages, including neurons and epithelial cells, but also endodermal lineages, including hepatocytes and muscle cells (14). The immune phenotypes of MSCs are positive expression of CD29, CD59, CD90 and CD105, but lack of expression of CD14, CD19, CD31 and CD34, and specifically, negative expression of the co-stimulators CD80, CD86 and HLA-E (15). MSCs also possess immunomodulatory properties, which enables them to suppress the activation and proliferation of T- and B-lymphocyte-mediated immune responses, as well as to interfere with the maturation and function of dendritic cells and natural killer cells (16). However, accumulating evidence indicated that the in vivo microenvironment is able to modify the low immune status of MSCs to finally result in an enhanced immune response $(7,8,9)$.

The TLR family is the most important class of signaling molecules associated with pathogen-associated molecular patterns and has a critical function in bridging innate and adaptive immune responses. In addition, TLR ligands have been linked with the perpetuation of inflammation in a number of chronic inflammatory diseases due to the permanent presentation of the immune system with TLR-specific stimuli (17). A previous study indicated that the activation of numerous TLR-associated pathways did not alter the immune status of MSCs (11); however, the study used MSCs isolated from $\mathrm{BM}$. In recent years, the human UC has attracted increasing attention due to its increased efficiency with regard to the expansion, proliferation and differentiation potential of its MSCs. UCMSCs and BMMSCs show marked similarities in metabolic, biological regulatory and multicellular processes, although there are also differences between BMMSCs and UCMSCs, with the predominant genes in UCMSCs including neurogenesis transcriptional factors such as sex determining region Y-box 11 and paired-like homeodomain 1 (18).

Our group has investigated the role of TLRs in influencing the immune status of UCMSCs and has shown that a TLR7 agonist enhanced the immunogenicity of UCMSCs (19). The present study assessed the role of TLR5 in regulating the immune status and differentiation ability of UCMSCs. Among all TLR members, TLR5 locates on the cell surface, is responsible for the detection of flagellin and specifically recognizes the constant domain D1. The results of the present study indicated that activation of the TLR5 pathway did not increase the proliferation of PBMCs or the release of LDH in a
PBMC-UCMSC co-culture system in the presence of flagellin. Assessment of co-stimulatory molecules also showed that these were not affected by TLR 5 activation. Furthermore, TLR 5 had no influence or inductive effects on the differentiation ability of UCMSCs into adipocytes, osteoblasts or chondrocytes. These results indicated that activation of TLR5 did not affect the immune status and differentiation ability of UCMSCs. However, the present study detected an increased expression of pro-inflammatory molecules in UCMSCs upon flagellin treatment, which may indicate that the induced immune-associated factors have biological functions in UCMSCs other than those assessed in the present study, which will be addressed in a future study.

\section{Acknowledgements}

The present study was supported by grants from the National Natural Scientific Foundation of China (no. 81200315) and the China Postdoctoral Science Foundation (nos. 2011M501413 and 2013T60855).

\section{References}

1. Bordignon C, Carlo-Stella C, Colombo MP, De Vincentiis A, Lanata L, Lemoli RM, Locatelli F, Olivieri A, Rondelli D, Zanon $\mathrm{P}$ and Tura S: Cell therapy: Achievements and perspectives. Haematologica 84: 1110-1149, 1999.

2. Phinney DG and Prockop DJ: Concise review: Mesenchymal stem/multipotent stromal cells: The state of transdifferentiation and modes of tissue repair-current views. Stem Cells 25: 2896-2902, 2007.

3. Bassi E, Aita CA and Câmara NO: Immune regulatory properties of multipotent mesenchymal stromal cells: Where do we stand? World J Stem Cells 3: 1-8, 2011.

4. Shi M, Liu ZW and Wang FS: Immunomodulatory properties and therapeutic application of mesenchymal stem cells. Clin Exp Immunol 164: 1-8, 2011.

5. Cui J, Wahl RL, Shen T, Fisher SJ, Recker E, Ginsburg D and Long MW: Bone marrow cell trafficking following intraveneous administration. Br J Haematol 107: 895-902.

6. Ankrum J and Karp JM: Mesenchymal stem cell therapy: Two steps forward, one step back. Trends Mol Med 16: 203-209, 2010.

7. Huang XP, Sun Z, Miyagi Y, McDonald Kinkaid H, Zhang L, Weisel RD and Li RK: Differentiation of allogeneic mesenchymal stem cells induces immunogenicity and limits their long-term benefits for myocardial repair. Circulation 122: 2419-2429, 2010

8. Nauta AJ, Westerhuis G, Kruisselbrink AB, Lurvink EG, Willemze R and Fibbe WE: Donor-derived mesenchymal stem cells are immunogenic in an allogeneic host and stimulate donor graft rejection in a nonmyeloablative setting. Blood 108: 2114-2120, 2006

9. Li Y and Lin F: Mesenchymal stem cells are injured by complement after their contact with serum. Blood 120: 3436-3443, 2012.

10. Blasius AL and Beutler B: Intracellular toll-like receptors. Immunity 32: 305-315, 2010.

11. DelaRosa O and Lombardo E: Modulation of adult mesenchymal stem cells activity by toll-like receptors: Implications on therapeutic potential. Mediators Inflamm 2010: 865601, 2010.

12. Peng Y and Zhang L: Activation of TLR1/2 pathway induced the shaping of immune response status of peripheral blood leukocytes. Exp Ther Med 7: 1708-1712, 2014.

13. Fu YS, Cheng YC, Lin MY, Cheng H, Chu PM, Chou SC, Shih YH, Ko MH and Sung MS: Conversion of human umbilical cord mesenchymal stem cells in Wharton's jelly to dopaminergic neurons in vitro: Potential therapeutic application for parkinsonism. Stem Cells 24: 115-124, 2006.

14. Tolar J, LeBlanc K, Keating A and Blazar BR: Concise review: Hitting the right spot with mesenchymal stromal cells. Stem cells 28: 1446-1455, 2010. 
15. Augello A, Kurth TB and DeBari C: Mesenchymal stem cells: A perspective from in vitro cultures to in vivo migration and niches. Eur Cell Mater 20: 121-133, 2010.

16. Han KH, Ro H, Hong JH, Lee EM, Cho B, Yeom HJ, Kim MG $\mathrm{Oh} \mathrm{KH}, \mathrm{Ahn} \mathrm{C}$ and Yang J: Immunosuppressive mechanisms of embryonic stem cells and mesenchymal stem cells in alloimmune response. Transpl Immunol 25: 7-15, 2011.

17. Akira S, Uematsu S and Takeuchi O: Pathogen recognition and innate immunity. Cell 124: 783-801, 2006.
18. Tomchuck SL, Zwezdaryk KJ, Coffel SB, Waterman RS, Danka ES and Scandurro AB: Toll-like receptors on human mesenchymal stem cells drive their migration and immunomodulating responses. Stem Cells 26: 99-107, 2008.

19. Zhang L, Liu D, Pu D, Wang Y, Li L, He Y, Li Y, Li L and Li W: The TLR7 agonist Imiquimod promote the immunogenicity of mesenchymal stem cells. Biol Res 48: 6, 2015. 